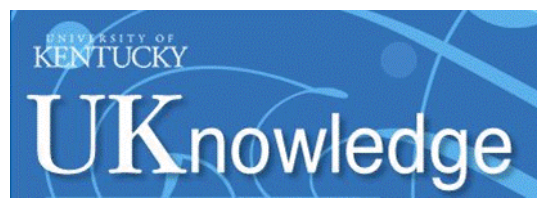

University of Kentucky

UKnowledge

\title{
$5-2015$
}

\section{Descriptive Analysis of Common Functional Limitations Identified by Patients with Shoulder Pain}

\author{
Enrique V. Smith-Forbes \\ University of Kentucky, e_vsf12@hotmail.com \\ Stephanie D. Moore-Reed \\ California State University - Fresno \\ Philip M. Westgate \\ University of Kentucky, philip.westgate@uky.edu \\ W. Ben Kibler \\ Shoulder Center of Kentucky \\ Timothy L. Uhl \\ University of Kentucky, tluhl2@uky.edu
}

Follow this and additional works at: https://uknowledge.uky.edu/rehabsci_facpub

Part of the Rehabilitation and Therapy Commons

Right click to open a feedback form in a new tab to let us know how this document benefits you.

\section{Repository Citation}

Smith-Forbes, Enrique V.; Moore-Reed, Stephanie D.; Westgate, Philip M.; Kibler, W. Ben; and Uhl, Timothy L., "Descriptive Analysis of Common Functional Limitations Identified by Patients with Shoulder Pain" (2015). Physical Therapy Faculty Publications. 46.

https://uknowledge.uky.edu/rehabsci_facpub/46

This Article is brought to you for free and open access by the Physical Therapy at UKnowledge. It has been accepted for inclusion in Physical Therapy Faculty Publications by an authorized administrator of UKnowledge. For more information, please contact UKnowledge@lsv.uky.edu. 


\section{Descriptive Analysis of Common Functional Limitations Identified by Patients with Shoulder Pain}

\section{Digital Object Identifier (DOI)}

http://dx.doi.org/10.1123/jsr.2013-0147

\section{Notes/Citation Information}

Published in Journal of Sport Rehabilitation, v. 24, no. 2, p. 179-188.

(c) 2015 Human Kinetics, Inc. as accepted for publication. http://dx.doi.org/10.1123/jsr.2013-0147 
In this era of evidence based medicine, clinicians have the responsibility to define

25 and measure the effect of treatment interventions. ${ }^{1}$ Decisions for further treatment

26 interventions and policies are based on the effectiveness of treatment outcomes. ${ }^{1}$

27 On January 1, 2013 the new Middle Class Tax Relief and Jobs Creation Act of 2012

28 (MCTRJCA; Section $3005(\mathrm{~g})$ ) took effect. ${ }^{2}$ A section of this new law requires health

29 care providers to collect data on Medicare patients' function during the course of

30 therapy services in order to better understand patient condition and outcomes. Therapy

31 services claims must now include non-payable G-code and related modifiers. The

32 MCTRJCA G-codes table for PT/OT claims-based functional reporting ${ }^{3}$ was designed to

33 incorporate G-codes to define "functional limitations" synonymously with the

34 International Classification of Function (ICF) terminology "activity limitations and

35 participation restrictions". ${ }^{2}$ Insurance companies traditionally follow suit with Medicare

36 laws, and require rehabilitation therapists to provide goals with functional outcomes for

37 reimbursement purposes. Therefore, investigation on a younger population would likely

38 be beneficial, as the requirements will likely be expanded eventually to this patient

39 population.

Functional limitation reporting may have broader implications. Therapists can

41 benefit from use of a uniform language to describe activity and participation limitations

42 commonly reported by patients. The International Classification of Function (ICF) Health

43 model, adopted in 2001 by the World Health Organization (WHO), provides a framework

44 of common language with a scientific basis to measure health and health related

45 domains. ${ }^{4}$ The ICF has taxonomy of over 1400 categories, which are allotted to named

46 components in this bio-psycho-social model. The ICF classifies functioning within the 
47 domains of body functions (b), body structures (s), activities \& participation (d) and

48 environmental (e) and personal factors. ${ }^{4}$

49 Currently there is no standard self-report measure of shoulder function. A recent

50 systematic review on patient reported measures of shoulder pain conditions proposed

51 the use of a wide ranging condition-specific measure that captures assessments of

52 shoulder pain from a bio-psycho-social perspective. ${ }^{5}$ The Patient Specific Functional

53 Scale (PSFS) ${ }^{6}$ is designed to measure individual patient function and their progress in

54 a clinical setting. ${ }^{7}$ The patient reports the most important functional activities that are

55 limited as result of their injury and it is not condition specific. The PSFS is particularly

56 suited to measuring change in individual patients. ${ }^{7,8}$ However, this focus on individual

57 patient limitations can be perceived as a restriction of the PSFS. Although each patient

58 provides individual activity limitations there are many commonalities in the reported

59 limitations among patients with shoulder pain. Therefore, the purpose of this study was

60 to describe, from a patient's perspective, the most commonly expressed functional

61 limitation using the standardized terminology provided by the ICF model in patients

62 presenting with shoulder pain to a sports medicine orthopedic surgeon across different

63 diagnoses. These findings will serve to help clinicians focus their assessment and

64 interventions on the primary shoulder functional limitations.

Methods

67 Participants

68 The data for this descriptive study were obtained from another prospective cohort

69 study of patients with shoulder pain presenting to a sports medicine orthopedic 
70 surgeon's office..$^{9}$ The data presented in this study are a secondary analysis of data

71 collected to examine factors that predict outcome of patients with suspected superior

72 labral injuries. ${ }^{9}$ All volunteers provided written consent prior to participation approved by

73 the University of Kentucky and Lexington Clinic Institutional Review Boards.

74 Patient recruitment criteria were established a priori. Since the target was not for

75 full thickness tears and we expected patients older than 60 years to present differently,

76 patients were recruited to participate in this study if they were between 15 and 60 years

77 of age, reported pain with overhead activity, and presented with a clinical history

78 consistent with dysfunction due to musculoskeletal shoulder injury (Figure 1). Patients

79 were excluded if they reported numbness and tingling in the upper extremity, as well as

80 symptoms and signs consistent with: 1) Cervical radiculopathy ${ }^{10}$ (positive upper limb

81 tension test, positive spurling test, relief of symptoms with distraction test, limited

82 cervical rotation $<60^{\circ}$ to side of discomfort). 2) Adhesive capsulitis, ${ }^{11}$ (no or only trivial

83 shoulder trauma, marked loss of active and passive shoulder motion in external rotation,

$84 \geq 50 \%$ especially with shoulder abducted to $90^{\circ}$, pain at the extremes of all motions,

85 globally limited glenohumeral translation, normal findings on true anteroposterior and

86 axillary radiographs of the shoulder). 3) Glenohumeral arthritis, ${ }^{12}$ (radiographic

87 evidence of joint space narrowing and/or osteophyte formation, crepitus observed with

88 shoulder motion, reported history of osteoarthritis). 4) History of osteoarthritis, steroid

89 injection within the last month or, surgery on the involved shoulder within the previous

90 year.

91176 participants were examined by a single sports medicine orthopedic surgeon

92 using standardized physical examination and history to be included. These176 
93 participants were further sub-divided into 4 categories to identify if functional limitations

94 differed between patients suspected to a clinical diagnosis consistent with superior

95 labral anterior - posterior (SLAP) lesions $(n=59)$, sub-acromial impingement (SAI) pain

96 ( $n=47)$, combined findings of both SLAP and SAI $(n=22)$, and non-specific shoulder pain

$97(\mathrm{n}=48)$. The demographical data is presented in Table 1.

$98 \quad$ Clinical exam inclusion criteria were derived based on previous cluster

99 examination approach for making a clinical diagnosis for superior labral and

100 subacromial impingement. ${ }^{13-17}$ Reliance was not placed on one exam or imaging test,

101 since no single test has been shown to be uniformly satisfactory to make the complete

102 diagnosis. ${ }^{18-20}$ A recent systematic review by Hegedus et. al. ${ }^{21}$ supports the concept of

103 using clusters of tests to make the clinical diagnosis in shoulder pathology.

104 For a patient to be categorized as having SLAP diagnosis required positive

105 findings in at least three of the following four clinical signs: history of popping or

106 catching, anterior slide maneuver, modified dynamic labral shear maneuver, ${ }^{15}$ active

107 compression test or a SLAP tear diagnosed by an MRI. For a patient to be categorized

108 as having SAI diagnosis required positive findings in at least three of the following five

109 clinical signs were positive: Neer test, Hawkins-Kennedy test, painful arc, Jobe test, and

110 weakness in external rotation. ${ }^{17}$ Patients that met both of these criteria were categorized

111 as combined SLAP \& SAI. Patients who had at least one positive finding for SLAP or

112 SAl criteria but did not meet either of the above criteria were categorized as non-

113 specific shoulder pain (Figure 1).

114 The 176 participants reported pain in their dominant shoulder the majority of the 115 time (146/176). Pain presented in the non-dominant arm much less frequently $(20 / 176)$ 
116 and 3/176 participants reported bilateral symptoms. Seven participants reported that

117 they were ambidextrous. Three participants had right side shoulder injuries, three had

118 left sided injuries and one participant had bilateral shoulder pain. Participants reported

119 the median pain and activity limitation duration of 6 months (range, 0.1-300 months).

$12051 \%$ of the injuries were caused by a traumatic event, and $15 \%$ of participants were

121 actively engaged in sports.

123 Procedure

124 At initial evaluation in the sports medicine orthopedic surgeon's office, patients 125 were asked to complete the Patient Specific Functional Scale (PSFS) with a member of 126 the research team. ${ }^{8}$ The PSFS has been found to be a valid, reliable, and responsive

127 outcome measure for patients with upper extremity problems. ${ }^{8}$ To complete the

128 questionnaire each patient was asked to identify 3-5 important activities that they were

129 unable to do or reported having difficulty with as a result of their shoulder problem.

130 Patients were also asked to rate their level of impairment from 0 to 10 for each activity 131 with 0 being "unable to perform activity" and 10 "able to perform activity at same level as

132 before injury or problem." The total PSFS score is generally reported as the average of

133 the scores. However, in this study, we focused on how patients scored each individual

134 activity. For example, if a patient reported a score of 2 when dressing themselves on

135 this scale this could be interpreted as $80 \%$ impairment in this task.

136 Members of the research team composed of a physical therapist/athletic trainer,

137 an athletic trainer and an occupational therapist/certified hand therapist, linked the

138 PSFS responses to the ICF. All three researchers had experience in treating patients 
139 with shoulder pain and were familiar with the ICF model. These researchers further

140 familiarized themselves with the established ICF linking rules process prior to starting

141 the study by reading three articles ${ }^{22-24}$ and met prior to starting the linking process to

142 review understanding of these rules and how to apply the rules to linking the PSFS to

143 the ICF. Fifteen sample cases were scored independently as previously described and

144 the investigators' results were discussed and consensus was determined prior to

145 starting data collection. ${ }^{22-24}$ According to the linking rules developed by Cieza et $\mathrm{al}^{23}$,

146 items from specific instruments can be linked to the best corresponding ICF categories,

147 and the representation of the ICF domains body functions (b), body structures (s),

148 activities \& participation (d) and environmental (e) and personal factors can be

149 examined. Following these rules, meaningful concepts within each item of the PSFS

150 were first identified before starting the linking process to ICF categories. ${ }^{23}$ The ICF rules

151 were followed to link meaningful concepts to one or more ICF categories to the third

152 level in order to maximize category representation per diagnoses. For example, "I have

153 difficulties pitching a baseball" contains 2 meaningful concepts: pitching and baseball.

154 Pitching was linked to hand and arm use (d445) and baseball was linked to recreation

155 and leisure (d920) of the ICF model. In cases when a response could not be interpreted

156 or could not be linked to one of the 1400 ICF components, the non-definable option "nd"

157 was used to link concepts not clearly specified.

158 The overall process of linking meaningful concepts to the ICF was done in an

159 iterative manner. ${ }^{25,26}$ The three researchers came together after independently

160 reviewing and linking meaningful concepts. The agreement between the researchers at

161 each level is presented as percent agreement ${ }^{26}$ in Table 2. It should be noted that 
162 about one quarter (24-27\%) of non-agreement cases occurred when one rater assigned

163 additional meaningful concepts to a functional limitation that the other rater did not,

164 resulting in a comparison of one rater's response to another rater's lack of response.

165 When these instances are excluded, agreement at the chapter level improves to 94-

$16697 \%$. A final consensus was made at a meeting with all 3 researchers present and the

167 final decision was agreed upon as to which ICF category should be linked to the PSFS

168 identified concept. ${ }^{23-27}$ The consensus categorization is reported in the results.

170 Statistical Analysis

$171 \quad$ All data were entered into Microsoft Excel 2008 for Mac Version 12.3.4.

172 Descriptive analysis was performed using Stata 12.1 (Stata, College Station, TX).

173 Descriptive statistics were utilized to determine the frequency distributions of the linked

174 ICF codes. Comparison between the four diagnostic categories were carried out using

175 logistic regression models, which were fit using generalized estimating equations in

176 SAS version 9.3 (SAS Institute, Cary, NC) in order to account for the fact that some

177 subjects contributed multiple observations. The null hypothesis is that no differences

178 exist in the frequencies of reported functional limitations of the diagnostic categories.

179 This was only carried out for only the most frequently reported functional limitations of

180 sleep functions (b134), exercise tolerance (b455), lifting and carrying objects (d430),

181 hand and arm use (d445), and recreation and leisure activities (d920). There is

182 inadequate data to test this for the other functional limitations. Descriptive statistics of

183 mean and standard deviations were calculated for the severity of each functional

184 limitation reported on the PSFS score for each functional limitation by diagnostic

185 category. Five separate analysis of variance tests for each functional limitation listed 
186 above were carried out to test the null hypothesis that the reported severity level of the

187 functional limitation did not differ across the 4 diagnostic categories.

\section{Results}

176 participants reported 573 patient specific functional limitations. These

191 functional limitations yielded 765 meaningful concepts. The meaningful concepts were

192 linked to the ICF and divided per diagnosis as follows: SLAP $=255$, rotator cuff $=192$,

193 combined $=96$, and non-specific $=222$ as shown in Table 3 . The majority of the

194 meaningful concepts $634(83 \%)$ were linked to the activities and participation domain

195 while 129 (17\%) were linked to the body function domain. This distribution was similar

196 across all four diagnostic categories, with activities and participation representing

$197220(87 \%)$ for SLAP, $154(80 \%)$ rotator cuff, 80 (83\%) combined, and $180(81 \%)$ non-

198 specific shoulder pain. Nine out of a possible nine chapters in the domain of activity and

199 participation were represented in this sample of subjects. Three out of a possible eight

200 chapters of the body function were represented in this sample of subjects. Only two

201 reported functional limitations (0.26\%) ("repetitive motion" and "pressure with arm away

202 from body") were considered not definable (nd), due to lack of clarifying information.

203 The frequencies of the 26 specific functional limitation categories from the ICF identified

204 by patients with shoulder pain are presented in Table 3. The five most common

205 functional limitations reported by patients with shoulder pain accounted for 556/765

$206(72.7 \%)$ of all the functional limitations reported by patients presenting to a sports

207 medicine orthopedic surgeon for shoulder pain. The frequencies of reporting a

208 functional limitation was not different between the 4 diagnostic categories for the five 
209 most commonly reported functional limitations. Specifically, significant differences were

210 not observed with respect to: sleep functions $(P=.71)$, exercise tolerance $(P=.26)$,

211 lifting and carrying objects ( $\mathrm{P}=.91)$, hand and arm use $(\mathrm{P}=.88)$, and recreation and

212 leisure activities $(P=.34)$. Furthermore, comparison of the severity of functional

213 limitations did not differ between the 4 diagnostic categories for the five most common

214 functional limitations examined: sleep functions $(P=.28)$, exercise tolerance $(P=.13)$,

215 lifting and carrying objects $(\mathrm{P}=.34)$, hand and arm use $(\mathrm{P}=.43)$, and recreation and

216 leisure activities $(P=.37)$. The descriptive analysis of the severity of functional limitation

217 for each diagnostic category is presented in Table 4. The average score on the PSFS at

218 the initial examination revealed that patients reported an overall average score of $4.0 \pm$

2192.5 out of 10 points on the PSFS. There was no difference in severity level on the PSFS

220 between the 4 diagnostic categories $(P=.27)$.

\section{Discussion}

We used the ICF as a reference to identify, categorize, and quantify meaningful

224 concepts extracted from individualized PSFS of patients seeking care from a sports

225 medicine orthopedic surgeon for shoulder pain. The purpose of this study was to help

226 clinicians identify common functional limitations in assessment and identify for treatment

227 interventions. Although patients present with several individualized functional limitations,

228 the findings of this study indicate that there is much similarity between patients and

229 across diagnostic categories. Our findings showed that patients presenting to a sports

230 medicine orthopedic surgeon with shoulder present with a large number of limitation

231 with daily activities and relatively few limitations with body functions. Although each 
232 patient reports many specific individual functional limitations, these results support that

233 there is much commonality between patients' functional limitations as five primary

234 functional limitations represented by the ICF codes accounted for $73 \%$ of all reported

235 limitations. The five categories are; Hand and arm use (d445) is defined as performing

236 the coordinated actions required to move objects or to manipulate them by using hands

237 and arms, such as when turning door handles or throwing or catching an object. ${ }^{28}$,

238 Lifting and carrying objects (d430) is defined as raising up an object or taking something

239 from one place to another, such as when lifting a cup or carrying a child from one room

240 to another. ${ }^{28}$ Exercise tolerance functions (b455) is defined as functions related to

241 respiratory and cardiovascular capacity as required for enduring physical exertion. ${ }^{28}$

242 Recreation and leisure activities (d920) is defined as engaging in any form of play,

243 recreational or leisure activity, such as informal or organized play and sports, programs

244 of physical fitness, relaxation, amusement or diversion, going to art galleries, museums,

245 cinemas or theatres; engaging in crafts or hobbies, reading for enjoyment, playing

246 musical instruments; sightseeing, tourism and travelling for pleasure. ${ }^{28}$ Sleep function

247 (b134) is defined as general mental functions of periodic, reversible and selective

248 physical and mental disengagement from one's immediate environment accompanied

249 by characteristic physiological changes. Although these descriptions are broad using

250 the ICF definitions they provide clinicians a more focal starting point in both identifying

251 and treating functional limitations.

252 Our findings revealed that patients have many activity and participation

253 limitations and these limitations are more prevalent than body function limitations. This

254 is consistent with a recent systematic review that investigated outcome measures used 
255 for shoulder pain patients. ${ }^{5}$ The measures included more than twice as many concepts

256 of activities and participation than concepts of body functions and structures. ${ }^{5}$ Our

257 results suggest that patients are primarily interested in activities that they cannot

258 perform. Our findings further support this study, and that many of the shoulder outcome

259 measures are appropriately framed as they tend to have many questions that focus on

260 activities and participation. ${ }^{5}$ Similar results were obtained in a recent study investigating

261 the extent to which patient generated PSFS items reflect ICF domains. ${ }^{29}$ In that study,

262 the upper limb represented $20 \%$ of the 2911 total items, where the ICF's activity and

263 participation component had strong representation (87.6\%), and weak representation of

264 body structures and function $(6.2 \%) .{ }^{29}$

265 Limitations to activities and participation are an important component when

266 assessing shoulder function. However, other researchers have noted that, in general,

267 clinicians are more inclined to use outcome measures of impairment such as pain and

268 range of motion. ${ }^{30,31}$ One significant drawback of PSFS is that limitations are

269 individualized. The findings of the current study suggest that many of these

270 individualized responses can be grouped together under the more standardized ICF

271 terminology. By grouping limitations in this manner we can more clearly describe or

272 characterize a patient with particular limitations with the same anatomical lesion. For

273 example, we had two patients categorized as having signs and symptoms consistent

274 with a SLAP lesion. One patient was 18 years old and reported difficulty lifting groceries

275 (lifting and carrying) and throwing a ball (hand and arm use) while the other patient was

27635 years old and reported difficulty with sleeping on his shoulder (sleeping function),

277 scratching his back (caring for body part), and doing push-ups (exercise tolerance). 
278 These descriptors may eventually lead to more specific and focused treatment

279 interventions based on the described limitations. Based on our results, we agree with

280 Fairbairn et $\mathrm{al}^{29}$ that the PSFS would complement impairment measures by

281 representing activity and participation components. In the current study $86 \%$ of all

282 patient reported functional limitations coded into meaningful concepts were represented

283 by 10 ICF codes. Therefore, to help standardize this reporting we have provided

284 clinicians with a proposed checklist derived from the most common activity and

285 participation limitations identified in this sample of patients with shoulder pain.

286 (Appendix 1).

287 The current study contributes unique information, in that regardless of suspected

288 diagnosis, functional limitations did not differ by frequency or by severity. With the high

289 functional demands placed on the shoulder during everyday life ${ }^{32}$ the functional

290 limitations would be similar regardless of the anatomical diagnosis. A primary goal of

291 any intervention is to return a patient to their normal level of function. Our clinical

292 experience is consistent with these results that by finding a position of comfort to sleep

293 and figuring out a way to allow a patient to lift their arm or lift up an object with less pain

294 are consistent across multiple pathologies.

295 The overall level of dysfunction was a bit more surprising as we found no

296 differences across suspected diagnoses. This is perhaps due to our sampling of

297 subjects seeking care from a sports medicine orthopedic surgeon that have perhaps

298 seen other health care providers and tried previous intervention prior to seeking the

299 advice of orthopedic surgeon. This is further supported by the overall level of

300 dysfunction was rated a 4 out 10 on the PSFS scale indicating that the patient were 
301 functioning at $40 \%$ of normal which is quite dysfunctional. This is consistent with the

302 literature of other patients seeking medical care. Patients with rotator cuff impingement

303 reported similar levels of impairment using the PSFS, although in that study three

304 activities were chosen for the PSFS. ${ }^{33}$ While in the current study $3-5$ activities were

305 utilized as recommended by the PSFS creators. ${ }^{6}$ The authors summed the PSFS

306 scores and recorded a median score of 13, which equals 4.3 if the 3 activities were

307 divided. ${ }^{34}$ This is quite comparable to the current study's findings of 3.5 level of shoulder

308 impairment. The clinical implication of this finding is that clinicians can expect patients to

309 present with moderate to high levels of impairment prior to seeking care.

\section{$311 \quad$ Limitations}

This sample represents individuals seeking medical care from a single sports

313 medicine orthopedic surgeon in one clinic over a period of two years and may not

314 generalize to the rest of the population of the US with other types of shoulder disorders.

315 The data for this study were obtained from a cohort study that had specific inclusion and

316 exclusion criterion that are stated previously; therefore caution must be applied when

317 generalizing these findings to other patients with shoulder pain that were excluded.

318 Although there were differences between the mean age of our sample (40 \pm 12 years)

319 and the average Medicare recipient's age (65+ years), this study helps to fill a vacuum

320 on the understanding of the most common limitations in patients with shoulder pain.

321 Specific pathoanatomical diagnoses were not confirmed with additional diagnostic

322 imaging for all patients therefore the categorized diagnosis may be incorrect. We

323 attempted to us a cluster of tests to categorize patients to the best of our ability 
324 however; there were a substantial number of patients not meeting the specific criterion

325 necessitating the development of the $4^{\text {th }}$ category on non-specific shoulder pain. There

326 is the potential for recall bias for symptom intensity as patients may have favored

327 positive memories more than negative ones. ${ }^{35}$ Although there were similarities in our

328 results with that of other researchers, the methods of researcher agreement might yield

329 different results with other groups if replicated. Future investigators may consider

330 performing and discussing additional sample cases prior to initiating the linking process

331 to increase agreement. Although our results appear as a lower level of ICF coding

332 agreement, as stated above, one quarter of non-agreement cases occurred when rater

333 assigned additional meaningful concepts to a functional limitation that the other did not.

334 Excluding these instances, agreement at the chapter level improves to $94-97 \%$.

\section{Conclusion}

This study demonstrated that individual functional limitations from a group of

338 patients could be clearly categorized using the ICF taxonomy. Approximately $51 \%-65 \%$

339 of four shoulder conditions: shoulder anterior labral tear from anterior to posterior

340 (SLAP), rotator cuff, combined SLAP and rotator cuff, and non-specific, of all functional

341 limitations identified by 176 patients could be represented by 5 ICF categories: Lifting

342 and carrying objects, Hand and arm use, Exercise tolerance, Sleeping Functions, and

343 Recreation and Leisure activities. Further, this study demonstrated that although

344 patients reported 573 different functional limitations these could be condensed into 26

345 specific categories using the ICF taxonomy. Ten of these categories represented $86 \%$

346 of all functional limitations reported by patients suspected to either have a SLAP lesion, 
347 sub-acromial impingement, a combination of both SLAP and sub-acromial impingent, or

348 non-specific shoulder pain. These patients presenting to a sports medicine orthopedic

349 surgeon on average consider themselves $60 \%$ functionally impaired, which represents 4

350 out of 10 points on the PSFS. This information should help health care professionals

351 focus on evaluating and treating the primary functional limitations that patients with

352 shoulder pain are likely to present on their initial visit. 
354

355

356

357

358

359

360

361

362

363

364

365

366

367

368

369

370

371

372

373

374

375

376

377

378

379

380

381

382

383

384

385

386

387
1. McMillan CR, Binhammer PA. Which outcome measure is the best? Evaluating responsiveness of the Disabilities of the Arm, Shoulder, and Hand Questionnaire, the Michigan Hand Questionnaire and the Patient-Specific Functional Scale following hand and wrist surgery. Hand (N Y). Sep 2009;4(3):311-318.

2. U.S. Department of Health and Human Services. Federal Register 42 CFR Parts 410, 414, 415, et al. In: Services CfMM, ed. Vol 77 2012:68891-69380.

3. U.S. Department of Health and Human Services. Federal Register 42 CFR Parts 410, 414, 415, et al. In: Services CfMM, ed. Vol 772012:68966-68967.

4. International Classification of Functioning DaH. World Health Organization. 2003.

5. Roe Y, Soberg HL, Bautz-Holter E, Ostensjo S. A systematic review of measures of shoulder pain and functioning using the International classification of functioning, disability and health (ICF). BMC musculoskeletal disorders. 2013;14(73):1-12.

6. Stratford P, Gill C, Westaway M, Binkley J. Assessing disability and change on individual patients: A report of a patient specific measure. Physiotherapy Canada. 1995;47(4):258-263.

7. Westaway MD, Stratford PW, Binkley JM. The patient-specific functional scale: validation of its use in persons with neck dysfunction. The Journal of orthopaedic and sports physical therapy. May 1998;27(5):331-338.

8. Hefford $\mathrm{C}$, Abbott JH, Arnold R, Baxter GD. The patient-specific functional scale: validity, reliability, and responsiveness in patients with upper extremity musculoskeletal problems. The Journal of orthopaedic and sports physical therapy. Feb 2012;42(2):56-65.

9. Moore-Reed SD, Kibler WB, Sciascia AD, Uhl T. Preliminary Development of a Clinical Prediction Rule for Treatment of Patients With Suspected SLAP Tears. Arthroscopy : the journal of arthroscopic \& related surgery : official publication of the Arthroscopy Association of North America and the International Arthroscopy Association. Aug 142014.

10. Wainner RS, Fritz JM, Irrgang JJ, Boninger ML, Delitto A, Allison S. Reliability and diagnostic accuracy of the clinical examination and patient self-report measures for cervical radiculopathy. Spine. Jan 1 2003;28(1):52-62.

11. Griggs SM, Ahn A, Green A. Idiopathic adhesive capsulitis. A prospective functional outcome study of nonoperative treatment. The Journal of bone and joint surgery. American volume. Oct 2000;82-A(10):1398-1407.

12. Kelley MJ, Ramsey ML. Osteoarthritis and traumatic arthritis of the shoulder. Journal of hand therapy : official journal of the American Society of Hand Therapists. Apr-Jun 2000;13(2):148-162.

13. Walsworth MK, Doukas WC, Murphy KP, Mielcarek BJ, Michener LA. Reliability and diagnostic accuracy of history and physical examination for diagnosing glenoid labral tears. The American journal of sports medicine. Jan 2008;36(1):162-168. 
14. Michener LA, Doukas WC, Murphy KP, Walsworth MK. Diagnostic accuracy of history and physical examination of superior labrum anterior- posterior lesions. $J$ Athl Train. Jul-Aug 2011;46(4):343-348.

15. Ben Kibler W, Sciascia AD, Hester P, Dome D, Jacobs C. Clinical utility of traditional and new tests in the diagnosis of biceps tendon injuries and superior labrum anterior and posterior lesions in the shoulder. The American journal of sports medicine. Sep 2009;37(9):1840-1847.

16. Park HB, Yokota A, Gill HS, El Rassi G, McFarland EG. Diagnostic accuracy of clinical tests for the different degrees of subacromial impingement syndrome. The Journal of bone and joint surgery. American volume. Jul 2005;87(7):14461455.

17. Michener LA, Walsworth MK, Doukas WC, Murphy KP. Reliability and diagnostic accuracy of 5 physical examination tests and combination of tests for subacromial impingement. Archives of physical medicine and rehabilitation. Nov 2009;90(11):1898-1903.

18. Gobezie R, Zurakowski D, Lavery K, Millett PJ, Cole BJ, Warner JJ. Analysis of interobserver and intraobserver variability in the diagnosis and treatment of SLAP tears using the Snyder classification. The American journal of sports medicine. Jul 2008;36(7):1373-1379.

19. Kibler WB, Kuhn JE, Wilk K, et al. The disabled throwing shoulder: spectrum of pathology-10-year update. Arthroscopy : the journal of arthroscopic \& related surgery : official publication of the Arthroscopy Association of North America and the International Arthroscopy Association. Jan 2013;29(1):141-161 e126.

20. McFarland EG, Kim TK, Savino RM. Clinical assessment of three common tests for superior labral anterior-posterior lesions. The American journal of sports medicine. Nov-Dec 2002;30(6):810-815.

21. Hegedus EJ, Goode AP, Cook CE, et al. Which physical examination tests provide clinicians with the most value when examining the shoulder? Update of a systematic review with meta-analysis of individual tests. British journal of sports medicine. Nov 2012;46(14):964-978.

22. Silva Drummond A, Ferreira Sampaio R, Cotta Mancini M, Noce Kirkwood R, Stamm TA. Linking the Disabilities of Arm, Shoulder, and Hand to the International Classification of Functioning, Disability, and Health. Journal of hand therapy : official journal of the American Society of Hand Therapists. Oct-Dec 2007;20(4):336-343; quiz 344.

23. Cieza A, Geyh S, Chatterji S, Kostanjsek N, Ustun B, Stucki G. ICF linking rules: an update based on lessons learned. Journal of rehabilitation medicine : official journal of the UEMS European Board of Physical and Rehabilitation Medicine. Jul 2005;37(4):212-218.

24. Cieza A, Stucki G. The International Classification of Functioning Disability and Health: its development process and content validity. European journal of physical and rehabilitation medicine. Sep 2008;44(3):303-313.

25. Geyh S, Kurt T, Brockow T, et al. Identifying the concepts contained in outcome measures of clinical trials on stroke using the International Classification of Functioning, Disability and Health as a reference. Journal of rehabilitation 
medicine : official journal of the UEMS European Board of Physical and Rehabilitation Medicine. Jul 2004(44 Suppl):56-62.

26. Fayed N, Cieza A, Bickenbach JE. Linking health and health-related information to the ICF: a systematic review of the literature from 2001 to 2008. Disability and rehabilitation. 2011;33(21-22):1941-1951.

27. Coenen M, Cieza A, Stamm TA, Amann E, Kollerits B, Stucki G. Validation of the International Classification of Functioning, Disability and Health (ICF) Core Set for rheumatoid arthritis from the patient perspective using focus groups. Arthritis research \& therapy. 2006;8(4):R84.

28. WHO. ICF Browser. 2013; Internationa Classification of Function Browser. Available at: http://apps.who.int/classifications/icfbrowser/. Accessed November 17, 2013.

29. Fairbairn K, May K, Yang Y, Balasundar S, Hefford C, Abbott JH. Mapping Patient-Specific Functional Scale (PSFS) items to the International Classification of Functioning, Disability and Health (ICF). Physical therapy. Feb 2012;92(2):310-317.

30. Brockow $\mathrm{T}$, Cieza $\mathrm{A}$, Kuhlow $\mathrm{H}$, et al. Identifying the concepts contained in outcome measures of clinical trials on musculoskeletal disorders and chronic widespread pain using the International Classification of Functioning, Disability and Health as a reference. Journal of rehabilitation medicine : official journal of the UEMS European Board of Physical and Rehabilitation Medicine. Jul 2004(44 Suppl):30-36.

31. Copeland JM, Taylor WJ, Dean SG. Factors influencing the use of outcome measures for patients with low back pain: a survey of New Zealand physical therapists. Physical therapy. Dec 2008;88(12):1492-1505.

32. Largacha M, Parsons IMt, Campbell B, Titelman RM, Smith KL, Matsen F, 3rd. Deficits in shoulder function and general health associated with sixteen common shoulder diagnoses: a study of 2674 patients. Journal of shoulder and elbow surgery / American Shoulder and Elbow Surgeons ... [et al.]. Jan-Feb 2006;15(1):30-39.

33. Bernhardsson S, Klintberg IH, Wendt GK. Evaluation of an exercise concept focusing on eccentric strength training of the rotator cuff for patients with subacromial impingement syndrome. Clinical rehabilitation. Jan 2011;25(1):6978.

34. Young IA, Michener LA, Cleland JA, Aguilera AJ, Snyder AR. Manual therapy, exercise, and traction for patients with cervical radiculopathy: a randomized clinical trial. Physical therapy. Jul 2009;89(7):632-642.

35. Herrmann D. Reporting current, past, and changed health status. What we know about distortion. Medical care. Apr 1995;33(4 Suppl):AS89-94. 